\title{
Measurement of Group Velocity of 17.8-kc/s VLF Radio Waves
}

\author{
W. D. Westfall \\ U.S. Navy Electronics Laboratory, Radio Physics Division, San Diego, Calif., 92152
}

(Received Dec. 24, 1964; revised April 19, 1965)

\begin{abstract}
Group velocity of 17.8-kc/s VLF radio waves is determined by measuring the difference in time of arrival of the transmitted signals which travel over complementary great circle paths. The velocity is found to be $3.04 \times 10^{8}$ meters/second with an estimated error of approximately $\pm 0.10 \times 10^{8}$ meters/ second. Differences in measurement technique and general environmental conditions between this experiment and a previous measurement of 18-kc/s group velocity which was made in 1949 is discussed.
\end{abstract}

\section{Introduction}

\subsection{Equipment and Timing}

In a previous article [Westfall, 1964], a system for simultaneously measuring phase and amplitude of VLF radio waves over complementary great-circle paths was described. Sample data for the long and short great-circle paths were shown where the transmitter was NAA and the receiving site was at the U.S. Navy Electronics Laboratory at San Diego, Calif.

The long-path receiver "looks" for the long-path signal during the off periods of the short-path signal. The amount of time delay between the arrival of the short-path signal and the arrival of the long-path signal at San Diego may be used to measure the group velocity of the VLF signals. While this parameter can- not be determined pulse by pulse because the long-path signal is quite weak, a coherent detection system provides an integrated long-path signal voltage which will be a maximum when the delay between the arrival of the short-path signal and the "look time" on the longpath receiver is optimum.

Figure 1 is a block diagram of the long-path portion of the receiving system which was used to make the group velocity measurements.

The antenna system is composed of commercially available loop and whip antennas and appropriate circuitry to produce a cardioid receiving pattern with a null of about $30 \mathrm{~dB}$. This null is normally directed toward the transmitter along the short great-circle path. The VLF phase-tracking receiver is also of commercial design and provides an analog record of VLF phase. The phase coherent $1 \mathrm{kc} / \mathrm{s} I F$ is passed

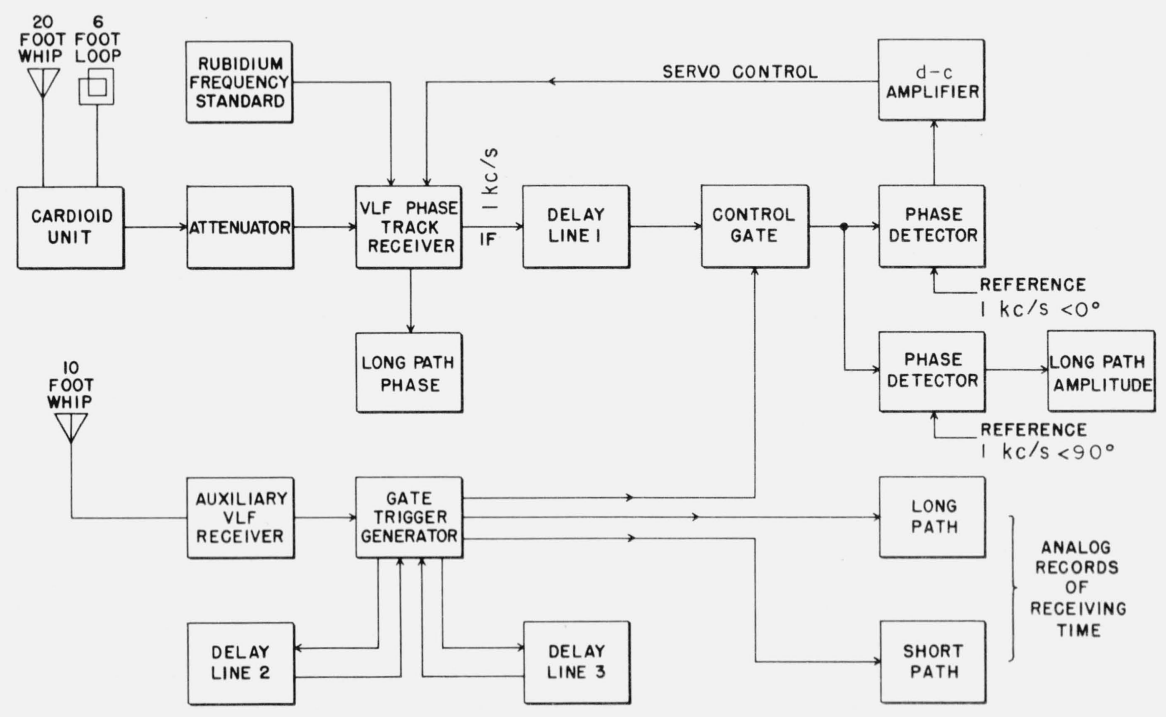

FIGURE 1. VLF receiving system designed to reject the short-path signal and measure phase and amplitude of the long-path signal. 
through delay line 1 and enters the gating circuits. The gating circuits are controlled by the VLF signal received via the short path on the auxiliary receiver. The gates are normally turned on only when the short path signal is off and also only at the approximate time of arrival of the long-path signal. This timing arrangement is accomplished by conventional pulse circuitry in the gate trigger-generator.

Figure 2 shows this timing in somewhat idealized detail. Because of the slope in the leading and trailing edges of the short-path signal, Pulse A is narrower than the signal which produced it. The trailing edge of Pulse B is delayed by delay line 2 for a time interval marked $\tau_{2}$. The short-path signal is delayed by delay line 1 for a time interval marked $\tau_{1}$ until it coincides with Pulse B. During Pulse B, the receiver is turned off by the control gate. Pulse $\mathrm{A}$ is also delayed by delay line 3 until it coincides with the arrival of the long-path signal. At this time, the control gate turns the receiver on if no short-path signal is present. In this example, the short-path signal is so long that the long-path receiving gate is shorter than the long-path signal and only a portion of the long-path signal is received.

The gate trigger generator also provides analog voltages which give the percent of time that the short-path signal is present and also the percent of time that the long-path receiver looks for the longpath signal.

The integrated long-path amplitude voltage must be normalized because the look times are at random periods and the amount of time that the long-path receiver is actually receiving long-path signal will vary depending upon the nature of the code transmissions. The percentage of time that the long-path

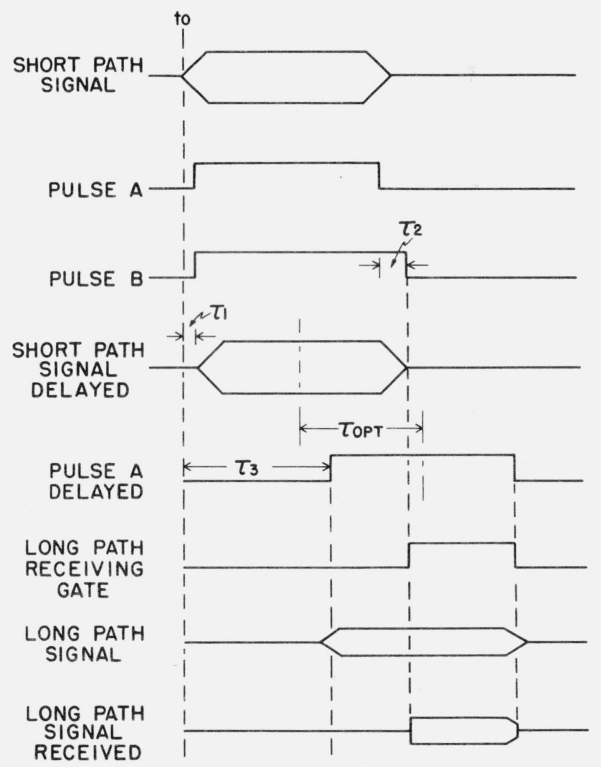

FIGURE 2. Long-path VLF receiver timing diagram. receiver is turned on is recorded as an analog voltage, and the normalized voltage $V_{n}$ is defined as

$$
V_{n}=\frac{V}{D}
$$

where $V$ is the integrated long-path signal voltage and $D$ is the percentage of time that the long-path receiver is turned on.

The delay time between short-path signal arrival and long-path "look" can be adjusted from zero to nearly $200 \mathrm{msec}$ and an optimum arrival time for the long-path signal may be determined.

\section{Uncertainties Contributing to Measure- ment Error}

While the experiment appears to be simple, several factors combine to make the determination of a precise value for group velocity quite difficult, i.e.,

(1) The random nature of code transmissions makes it difficult to determine precisely the average shape of the short-path keyed characters. It is necessary to know this shape in order to define a zero time.

(2) The long-path signal amplitude may be constantly fluctuating in an unpredictable manner, which reduces the significance of any single set of consecutive readings.

(3) The amount of atmospheric noise, short-path signal amplitude, and subtle changes of wave shape in the short-path signal may influence the operation of the timing gate generator used in this system.

(4) The delays inherent in the long-path receiver and the control receiver which provides signal to operate the timing gates are not the same and may not be constant under all conditions.

(5) Finally, the long-path receiving gate is somewhat shorter than the short-path signal which produces it. This has the effect of broadening the period of optimum time delay.

\section{Experimental Procedure and Results}

Figure 3 shows an estimate of the smoothed response curve throughout a $200 \mathrm{msec}$ delay range. The data points were obtained by delaying pulse A (fig. 2) with respect to the time of arrival of the short-path signal. This figure is the result of about 400 individual readings taken during different hours of the day on 17 days. Since the attenuation rate changes with time, the integrated amplitude voltage of the long-path signal varies with time, and as a result $V_{n}$ varies widely for the different sets of data. The value of $V_{n}$ which was nearest to $104 \mathrm{msec}$ was made unity for each set and all values in the set were normalized accordingly. The response to small time delays is not shown because in this area $D$ is relatively small and small errors in $D$ produce large errors in $V_{n}$. At zero delay, $V$ equals zero. The data points in figure 3 suggest an optimum time delay, $\tau_{\text {opt }}$, of about 105 msec with an estimated uncertainty of $\pm 5 \mathrm{msec}$ which is due to the previously mentioned sources of experimental error. 
A calibration of the actual delay, which is equal to the quantity $\tau_{3}-\tau_{1}$ (fig. 2), was obtained by reversing the antenna cardioid pattern and photographing the short-path signal and the delayed pulse A while they were simultaneously displayed on a dual-beam oscilloscope. To determine the actual delay time for a given delay line setting, the delays between the leading edges as well as the trailing edges were measured. One half the sum of the averages of these delays represents the average delay $\tau_{\text {avg }}$ for a given delay line setting. Figure 4 shows the measured $\tau_{\text {avg }}$ for delay line settings of 110,120 , and 126 .

Further knowledge of the possible uncertainty of this curve may be obtained if it is assumed that the deviations in time of the leading and trailing edges from their respective averages is independent from the actual delay line settings, and also that the standard deviation of the leading and trailing edges is approximately equal to $\sqrt{\frac{\Sigma(\Delta \tau)^{2}}{n}}$ for either case, where $\Delta \tau$ is the deviation from the average and $n$ is the number of samples. For approximately 50 samples of each, the leading edge standard deviation was about 1.58 msec, and the trailing edge standard deviation was 1.55 msec. The combined standard deviation, $S$, which specifies the standard deviation for the overall delay is therefore $2.2 \mathrm{msec}$. From these considerations alone it appears that the line in figure 4 should have an upper and lower uncertainty of about 2 msec.

A second and perhaps more accurate method of determining $\tau_{\text {opt }}$ is to open the servo control circuit shown in figure 1 and to apply the quadrature d-c voltage outputs of the phase detectors to the vertical and horizontal inputs of an oscilloscope. The resulting deflection of the beam spot (which is a direct indication of amplitude voltage $V$ ) may be recorded with suitable time exposure photographs. Repeated measurements with different delay line settings around the estimated optimum time of arrival determine a range of optimum delay setting values which cannot easily be reduced.

On 26 February 1965 NAA departed from their usual FSK schedule and maintained unbroken code transmissions for several hours. This permitted a series of consecutive photographs to be made of both the circuit delay as well as the optimum time delay $\tau_{\text {opt }}$, thus improving the accuracy of this series of measurements. About 120 photographs were made in a period of $3 \mathrm{hr}$. The region in figure 4 marked "optimum range" is the result of the measurement technique just described. It is an area in which a maximum amplitude $V_{n}$ appeared to exist, but within which the point of peaking could not be exactly determined. From figure 4 it is concluded that $\tau_{\text {opt }}$ was $102 \pm 3.5$ msec.

If it is assumed that the group velocity over the first 4.5 megameters $(\mathrm{Mm})$ of the long path is the same as that over the short path (which is $4.5 \mathrm{Mm}$ long), the optimum time delay measurement applies to a path $31.0 \mathrm{Mm}$ long and the resulting computed group velocity is $3.04 \times 10^{8} \mathrm{~m} / \mathrm{sec}$ with an estimated error

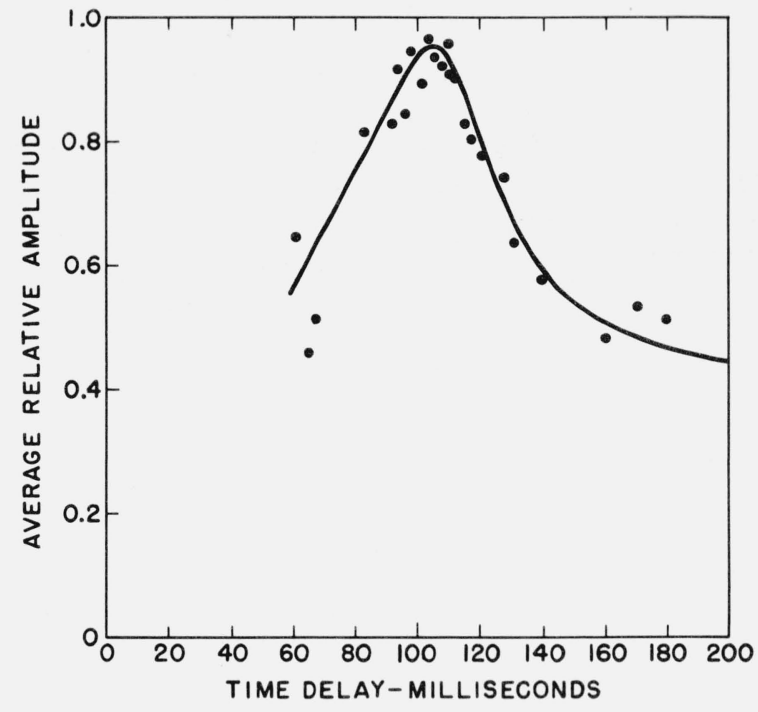

FigURE 3. Average relative amplitude as a function of time delay.

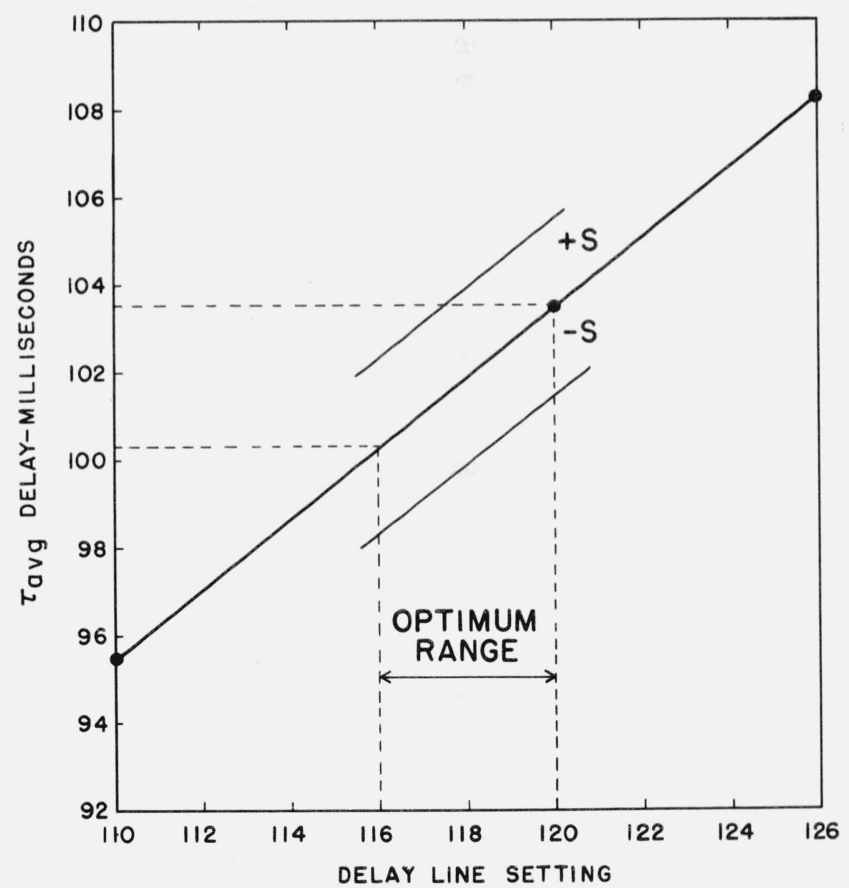

FIGURE 4. Measured actual delay as a function of delay line setting and measured optimum range for maximum $\mathrm{V}_{\mathrm{n}}$.

of $\pm 0.10 \times 10^{8} \mathrm{~m} / \mathrm{sec}$. If for physical reasons group velocities greater than the speed of light are rejected, the final limits applying to this measurement would be $2.94 \times 10^{8} \mathrm{~m} / \mathrm{sec}$ to $3.0 \times 10^{8} \mathrm{~m} / \mathrm{sec}$.

From published theoretical curves for phase velocity [Wait and Spies, 1964] it appears that there is little difference between east-west and west-east daytime propagation at $17.8 \mathrm{kc} / \mathrm{s}$ and therefore the 
group velocity would not be expected to differ appreciably for the two directions. Also, experimental data at $10.2 \mathrm{kc} / \mathrm{s}$ [Pierce and Nath, 1961] shows that the daytime phase velocities over land and sea differ by only about 0.05 percent which suggests that the travel time of the radio waves would not differ appreciably for the two $4.5-\mathrm{Mm}$ paths.

If a group velocity $V_{g}$ is expressed as

$$
V_{g}=\frac{V_{p}^{2}}{\left[V_{p}-f \frac{\left(d V_{p}\right)}{d f}\right]}
$$

where $f$ is frequency and $V_{p}$ is phase velocity, the phase velocity curves of Wait and Spies [1964] may be used to obtain a theoretical group velocity at this frequency of about $2.98 \times 10^{8} \mathrm{~m} / \mathrm{sec}$. It should be noted, however, that both $V_{p}$ as well as the derivative of phase velocity with respect to frequency can change slightly with direction of propagation, ionospheric height, and ground conductivity.

This result agrees with the above measured value within the experimental error.

\section{Previous Group Velocity Measurement}

An early measurement of the group velocity of special 18-kc/s pulse transmissions was made by Brown [1949]. Round-the-world signals were observed at a site close to the transmitter at Annapolis, Md. A large loop was directed east-west and on days when the atmospheric noise was low, the long-path signals were directly visible on an oscilloscope and could be photographed. The group velocity determined in this way (assuming a $40 \mathrm{Mm}$ distance) is about $(2.91 \pm 0.01)$ $\times 10^{8} \mathrm{~m} / \mathrm{sec}$.

It is interesting to compare the conditions existing in 1949 with the conditions currently existing. It seems likely that the signals which Brown measured arrived primarily from the west because of antenna orientation and because attenuation rates for east-west and north-south propagation are greater. The NAA signal arrives from the west also. The $18-\mathrm{kc} / \mathrm{s}$ signals probably traveled over nearly the same path as the current NAA transmissions being measured at San
Diego except that the path over the continental United States added another $4.5 \mathrm{Mm}$ of land to the $18-\mathrm{kc} / \mathrm{s}$ path. The amount of land encountered on the long path divided by the total path length for the 18 and $17.8 \mathrm{kc} / \mathrm{s}$ measurements produce ratios of about 0.29 and 0.21 , respectively.

It should also be observed that the 1949 measurements were made during the peak of the 18th solar cycle when the smoothed sunspot number was about 130. The present solar activity is at minimum. A recent article [Poppoff et al., 1964] points out that the $D$-region structure is controlled by $\mathrm{x}$ radiation, cosmic rays, and Lyman-alpha, all of which change throughout the solar cycle.

The 18-kc/s measurements were made on 25 January and 8 March 1949 with a difference of approximately $0.8 \mathrm{msec}$ in delay being attributed to disturbed conditions on 25 January. The NAA group velocity data were obtained in October 1964 and February 1965. Seasonal effects are being observed in the NAA long path phase and amplitude records, but the manner in which these changes are related to possible group velocity changes must await further study.

The author is grateful to A. D. Watt and J. E. Bickel for helpful suggestions during the preparation of this material.

\section{References}

Brown, J. N. (1949), Round-the-world signals at very low frequency, J. Geophys. Res. 54, No. 4, 367-372.

Pierce, J. A., and S. C. Nath (1961), VLF propagation, Annual Progress Report No. 60, pp. 1-6, Cruft Laboratory, Harvard Univ. Poppoff, I. G., R. C. Whitten, and R. S. Edmonds (Oct. 1, 1964), The role of nonflare $\mathrm{X}$ radiation in the $D$ region, J. Geophys. Res. 69, No. 19, 4081-4085.

Wait, J. R., and K. P. Spies (Dec. 30, 1964), Characteristics of the earth-ionosphere waveguide for VLF radio waves, NBS Tech. Note. 300.

Westfall, W. D. (Nov. 1, 1964), Simultaneous measurement of phase and amplitude of NAA very low frequency east-west and westeast radio transmissions at San Diego, J. Geophys. Res. 69, No. 21, 4523-4529.

(Paper 69D9-557) 